\title{
ALICE forward rapidity upgrades
}

\author{
Maciej Slupecki* ${ }^{\dagger}$ \\ University of Jyvaskyla, Jyvaskyla, Finland \\ E-mail: maciej.slupecki@cern.ch
}

Two forward rapidity upgrades of the ALICE apparatus at the LHC are presented: the Muon Forward Tracker (MFT) and the Fast Interaction Trigger (FIT). Their designs are driven by physics performance studies, which have been done using MC simulations. Several prototypes have been tested. Currently the detector design phase has ended. The final layouts, geometries and expected performance figures are presented. The latest developments from the test of the prototypes are discussed.

The MFT will improve the performance of the ALICE Muon Spectrometer by adding vertexing capabilities to the system. The elementary component of the MFT is a Monolithic Active Pixel Sensor (MAPS), using the TowerJazz $0.18 \mu \mathrm{m}$ technology called ALPIDE. ALPIDE has been developed by the ALICE collaboration for upgrades of the Inner Tracking System and the MFT. The MFT will consist of five detection planes forming a cone-like structure located between the interaction point and the frontal absorber of the Muon Spectrometer. The quality of track matching between the MFT and the Muon Spectrometer has been evaluated using Monte Carlo simulations. The same simulations were also used to extract the pointing accuracy of reconstructed muon tracks, which defines the resolution of the reconstructed vertex position.

FIT has both online and offline functionalities. It will send online luminosity feedback to the LHC and it will generate minimum-bias and centrality-based triggers for ALICE. It is also expected to provide the offline information on the precise collision time for the Time-Of-Flight detector, as well as on forward multiplicity, centrality and event plane for $\mathrm{Pb}-\mathrm{Pb}$ collisions. It will be composed of two Cherenkov detector arrays, surrounding the beam pipe on both sides of the interaction point, and one scintillator ring. The arrays will use Micro Channel Plate (MCP-PMT) technology to detect Cherenkov light and sectors of the scintillator ring will be optically coupled with Fine Mesh PMTs. The arrays provide good time response, while the scintillator ring allows for larger active area coverage. The extensive simulations verify the performance of the detector in terms of centrality and event-plane resolution. Additionally, the test results of the prototype of a single Cherenkov detector module, installed in ALICE, are presented.

EPS-HEP 2017, The European Physical Society Conference on High Energy Physics

5-12 July 2017

Venice, Italy

\footnotetext{
* Speaker.

${ }^{\dagger}$ on behalf of the ALICE Collaboration
} 


\section{Introduction}

The Long Shutdown 2 (LS2) of the LHC collider at CERN is scheduled for 2019-2020. For the ALICE apparatus the most important changes implemented during LS2 will be the reduction of the bunch crossing time down to $25 \mathrm{~ns}$ for pp collisions and $50 \mathrm{~ns}$ for $\mathrm{Pb}-\mathrm{Pb}$ collisions, and the increase of the $\mathrm{Pb}-\mathrm{Pb}$ interaction rate up to $50 \mathrm{kHz}$. In order to fully exploit the increased $\mathrm{Pb}-\mathrm{Pb}$ luminosity, ALICE will improve many of its subsystems or replace them with new solutions [1]. Here we describe two new forward detector systems for the upgraded ALICE apparatus: the Muon Forward Tracker (MFT) and the Fast Interaction Trigger (FIT). Both detector systems cover the forward rapidity region of the ALICE apparatus (see Fig. 1).

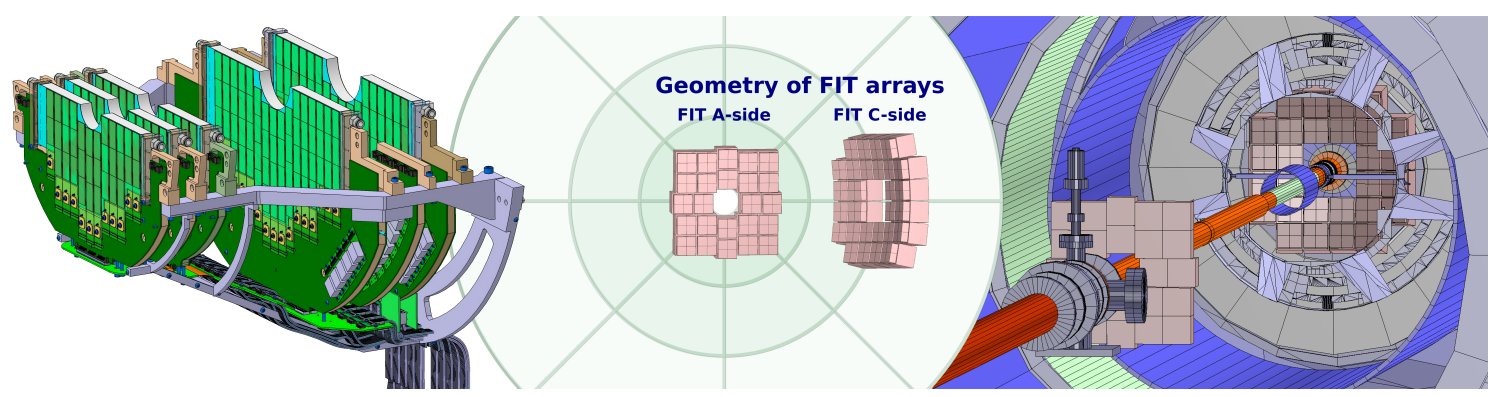

Figure 1: Left: Drawing of the bottom part of the MFT. Middle: Relative size of T0+ and V0+, the active components of FIT. Right: FIT arrays within ALICE, as seen in the simulation framework. For clarity, the MFT and V0+ scintillator ring on the A-side are not drawn.

\section{The Muon Forward Tracker Upgrade}

The MFT [2] will add vertexing capabilities to the Muon Spectrometer over a broad range of transverse momenta allowing ALICE to measure beauty down to $\mathrm{p}_{\mathrm{T}} \sim 0$ from displaced $\mathrm{J} / \psi$ vertices and to have an improved precision for the $\psi(2 S)$ measurement. It will also provide information on the forward multiplicity. The MFT will be located right after the Inner Tracking System (ITS), on the straight path between the interaction point (IP) and the frontal absorber of the Muon Spectrometer. The MFT will cover the pseudorapidity range of $-3.6<\eta<-2.5$. It will consist of five detector disks arranged in a cone-like structure. To facilitate the installation, each of the detector disks will be divided into half-disks (Fig. 1). The half-disks will be instrumented with ALPIDE sensors on both sides, ensuring more than $160 \%$ of redundancy. The first disk will be $460 \mathrm{~mm}$ away from the IP and the fifth, at a distance of $768 \mathrm{~mm}$. The disks are formed by 280 ladders, each containing 2-5 ALPIDE sensors. The total active area is $0.4 \mathrm{~m}^{2}$.

Since the MFT will be located in the confined volume, around the beam pipe, between the new ITS [3] and FIT detector, the design requirements are very stringent. In addition to the very restricted space envelope, the MFT detector has to be radiation hard, minimize material budget and implement sufficiently small pixel size to guarantee the desired tracking accuracy (spatial resolution of about $5 \mu \mathrm{m}$ ). Additionally, the readout of MFT must be capable of operating at a sustained interaction rate of $200 \mathrm{kHz}$ for pp and $50 \mathrm{kHz}$ for $\mathrm{Pb}-\mathrm{Pb}$ collisions. 


\subsection{ALPIDE sensor}

The elementary component of the MFT is a Monolithic Active Pixel Sensor (MAPS) using the TowerJazz $0.18 \mu \mathrm{m}$ technology, called ALPIDE. ALPIDE has been developed by the ALICE collaboration for the ITS and MFT upgrades. The pixel size is $27 \times 29 \mu \mathrm{m}^{2}$ and its thickness is $50 \mu \mathrm{m}$. A matrix of $1024 \times 512$ pixels forms a single ALPIDE chip with a size of $\sim 15 \times 30 \mathrm{~mm}^{2}$. The total material budget will be $0.7 \%$ of a radiation lenght per disk. The chip can withstand a radioactive dose of $300 \mathrm{krad}$, which is expected to accumulate close to the ALICE beam pipe during Run3 and Run4.

\subsection{Tracking performance}

Since the MFT is intended to expand the physics performance of the Muon Spectrometer by adding vertexing capabilities, the muon track matching probability between the MFT and the Muon Spectrometer is an important part of the feasibility studies of the overall system. By now two algorithms were investigated and found to result in similar percentage of correct track matches as a function of transverse momentum (see Fig. 2). This performance fully complies with the physics goals of the detector.

Another important feature, related to the aforementioned vertexing capabilities, is the pointing accuracy of the MFT. The resolution of the offset of the muon track from the interaction vertex is shown in Fig. 2: it ranges from about $100 \mu \mathrm{m}$ at low $\mathrm{p}_{\mathrm{T}}$ to $30 \mu \mathrm{m}$ at high $\mathrm{p}_{\mathrm{T}}$.
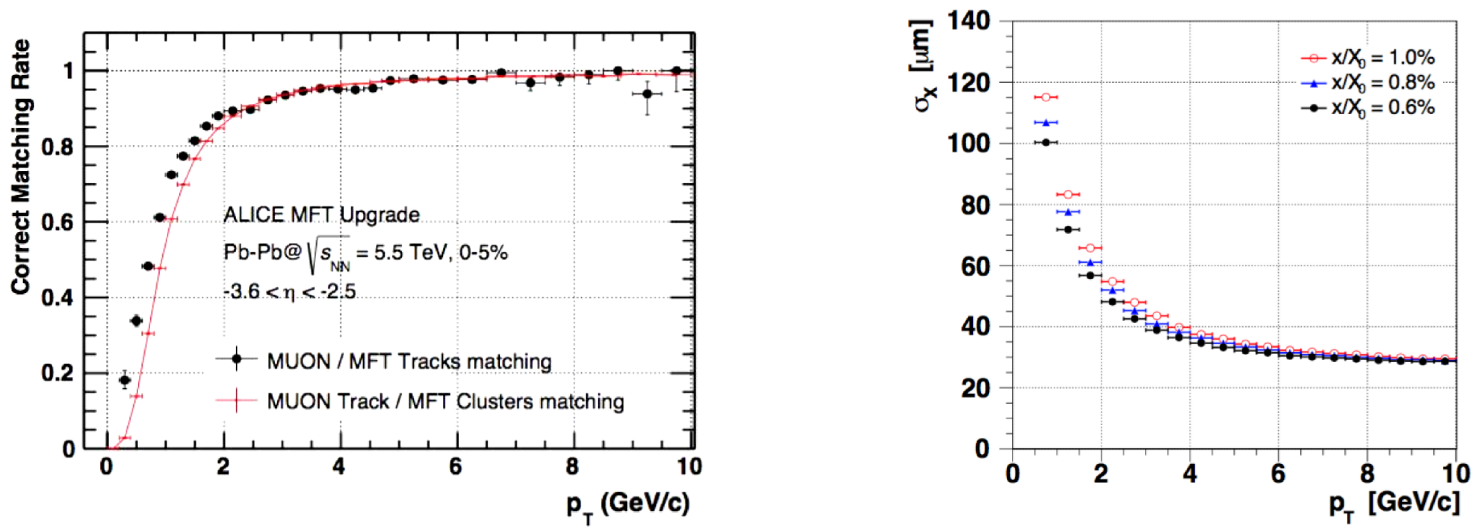

Figure 2: Left: Probability for correct track matching between the Muon Spectrometer and the MFT for two different algorithms. Right: Muon track transverse pointing accuracy as a function of transverse momentum for different material budgets.

\section{The Fast Interaction Trigger}

The FIT [4] is a multipurpose detector designed to provide both online and offline functionalities for the upgraded ALICE apparatus. The main online functionalities of FIT will be luminosity monitoring with a direct link to the LHC and the generation of a fast trigger signal for ALICE subsystems. The trigger must be generated with the latency of less than $425 \mathrm{~ns}$, of which $222 \mathrm{~ns}$ is 
the delay along the connecting cables. The trigger generated by FIT will allow for online vertex determination, minimum-bias and centrality-based event selection, suppression of beam-gas events, and a veto for ultra-peripheral collisions.

For the offline analysis, FIT will provide the precise collision time for the Time-of-Flight (TOF) based particle identification. This will be especially important in the case of low multiplicity events when the ALICE TOF detector registers too few trajectories for a precise reconstruction of the interaction time. FIT will also measure an unbiased multiplicity distribution in the forward direction, which is needed for the determination of the centrality and of the event plane in heavyion collisions.

\subsection{FIT design}

FIT is a hybrid detector composed of two Cherenkov detector arrays (T0+) and a large, sectored scintillator ring ( $\mathrm{V} 0+$ ). The T0+ arrays surround the beam pipe on both sides of the IP. The array on the Muon Spectrometer side is at the distance of $82 \mathrm{~cm}$ from the IP. On the opposite side, the array is at the distance of about $330 \mathrm{~cm}$ and it is supplemented by the scintillator ring V0+. In total the detector will be composed of 256 channels, including 48 scintillator signals and 208 Cherenkov signals, coming from 100 sensors.

\subsection{T0+}

The two Cherenkov arrays have a modular design. Each T0+ module is based on a PLANACON XP85015/A1-Q MCP-PMT light sensor optically coupled to four quartz radiators of $2.65 \times 2.65 \times 2 \mathrm{~cm}^{3}$ each. The anode of the PLANACON is also divided into four groups overlapping with the division of the radiator. Hence, each $\mathrm{T} 0+$ module acts as four independent detection channels. The modules are housed within a light-tight aluminum support. To facilitate installation, the support structure of each array is divided into two parts: top and bottom. The adjustment and calibration of the sensors will be done with a dedicated laser calibration system delivering $400 \mathrm{~nm}$ light pulses to each quadrant of every module. The light distribution system includes a picosecond laser, digital attenuator, optical fibers, splitters and micro-prisms concealed in the front covers of the modules. Precise determination of the delay of each detection channel is essential to reach the required time resolution of the array of better than $50 \mathrm{ps}$. Also the amplitudes of the signals have to be accurately adjusted to provide a reliable online multiplicity determination for the trigger. The expected dynamic range, especially for the modules closest to the beam pipe will be 1:300 as up to 300 minimum ionizing particles (MIPs) are expected per quadrant in central heavy-ion collisions.

\subsection{T0+ prototype tests}

A prototype of a single T0+ module has been operating inside of the ALICE setup since May 2016. It provides an opportunity to conduct tests in a real-life environment, which gives us a realistic evaluation of the performance of the detector, an assessment of the background and electronic interferences, and of the aging characteristics of the sensor. Figure 3 shows the influence of the magnetic field on the signal amplitude at different high-voltage settings. It can be noted, that the presence of the magnetic field of $0.5 \mathrm{~T}$ causes the gain of the MCP-PMT photosensor to 
decrease significantly. Figure 3 also presents the comparison of the time resolution of the T0+ module prototype $(<41 \mathrm{ps})$ and the currently used T0 detector $(\sim 42 \mathrm{ps})$. The prototype already complies with the requirement of $<50 \mathrm{ps}$. A photograph of the prototype, located within ALICE, is also shown in the Fig. 3.
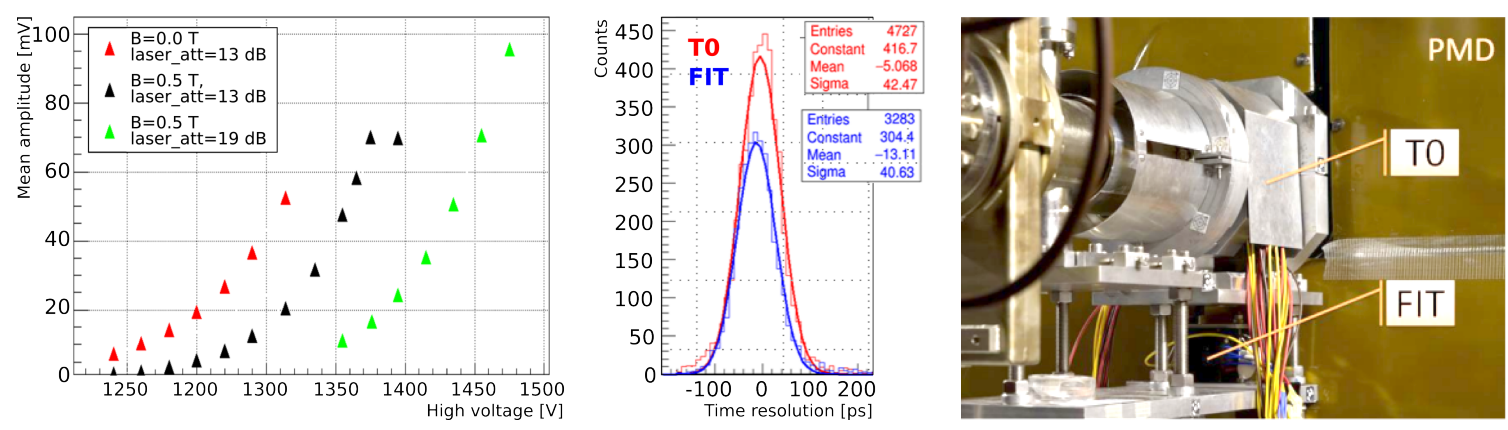

Figure 3: FIT T0+ prototype test results at ALICE. Right: A photograph of the T0 detector (aluminum cover) and the T0+ prototype (black box just below it) within ALICE. Left: MCP photosensor gain as a function of high voltage for different settings of the ALICE L3 solenoid magnet. Middle: Time resolution comparison between the current $\mathrm{T} 0$ detector and the $\mathrm{T} 0+$ prototype.

\section{$3.4 \mathrm{V0+}$}

Due to the limited space, the V0+ scintillator disk can be located only on one side of the IP. The outer diameter of the disk will be $148 \mathrm{~cm}$ providing a considerable increase in the acceptance as compared to the $\mathrm{T} 0+$ alone. The division of the scintillator into rings with gradually increasing width towards the outer edge reflects the expected distribution of charged particles. The size of the innermost four rings is based on the design of the current ALICE V0 detector [5]. For the determination of the reaction plane the $\mathrm{V} 0+$ detector needs to be divided radially into sectors. As a result of simulations and following the successful design of $\mathrm{V} 0$, we have split the $\mathrm{V} 0+$ disk radially into eight equal 45-degree sectors. The number of independent readout channels for $\mathrm{V} 0+$ will reach 48 , since, due to its size, each sector of the fifth ring needs two sensors. As the scintillator material we have chosen a $4 \mathrm{~cm}$ thick EJ-204. The surface of the scintillator will be viewed by clear optical fibers arranged on a 2D mesh with a pitch of $5 \mathrm{~mm}$. The fibers on the other end will be gathered into a bundle and coupled to a 2-inch fine mesh PMT R5924-70 by Hamamatsu. The length of the fibers within one sector will be equalized.

$\mathrm{V} 0+$ will use the same readout electronics as $\mathrm{T} 0+$.

\subsection{Physics performance simulations}

In addition to tests of prototypes with MIPs from the Proton Synchrotron at CERN, the performance of FIT has been verified by extensive MC simulations. In particular, we have evaluated FIT performance in terms of centrality and event-plane resolution. Figure 4 summarizes the results. The Cherenkov arrays ( $\mathrm{T} 0+$ ) alone would show inadequate centrality and event-plane resolution. The addition of a large scintillator ring ( $\mathrm{V} 0+)$ improves the performance on the A-side, where it is located. On the other side (C-side) the MFT is present, which together with FIT will provide comparable, or better performance to that of the current ALICE V0 system. 

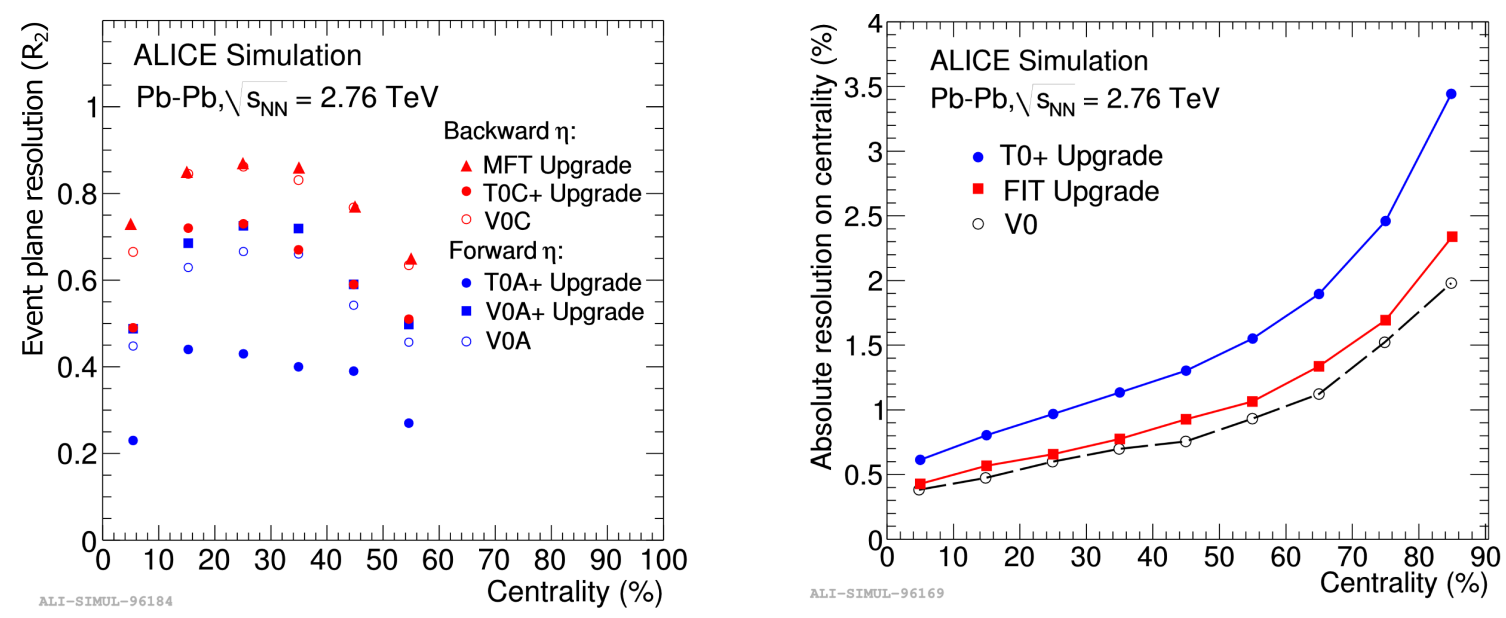

Figure 4: Simulation results of the event-plane reconstruction resolution (left) and centrality resolution (right) as a function of centrality.

\section{Summary}

The design of the MFT and the FIT detector systems is nearly completed as both detectors are entering the production stage. Beam tests and simulations confirm that the detectors meet the design parameters and thus will make a significant contribution to the physics programme of the upgraded ALICE apparatus during Run3 and Run4 of the LHC.

\section{Acknowledgements}

This work was partially supported by the Helsinki Institute of Physics, the projects PAPIITUNAM IN-111117 and CONACYT 280362.

\section{References}

[1] B. Abelev et al. Upgrade of the ALICE Experiment: Letter Of Intent. J. Phys., G41:087001, 2014.

[2] J. Adam et al. Technical Design Report for the Muon Forward Tracker. Technical Report CERN-LHCC-2015-001. ALICE-TDR-018, CERN, Jan 2015.

[3] B. Abelev et al. Technical Design Report for the Upgrade of the ALICE Inner Tracking System. Technical Report CERN-LHCC-2013-024. ALICE-TDR-017, CERN, Nov 2013.

[4] Wladyslaw Henryk Trzaska. New Fast Interaction Trigger for ALICE. Nucl. Instrum. Meth., A845:463-466, 2017.

[5] E. Abbas et al. Performance of the ALICE VZERO system. JINST, 8:P10016, 2013. 